\title{
Diffraction of SH Waves by an Elliptic Inclusion with Partially Debonded Region in Bi-Material Half Space
}

\author{
Ding xiaohao, Qi hui \\ College of Aerospace and Civil Engineering,Harbin Engineering University,Harbin, China
}

\begin{abstract}
Keywords: bi-material half space; SH waves; partially debonded region; comformal mapping
Abstract: Base on elastodynamics, complex function method with mapping function and Green's function method are used to investigate the scattering of SH waves by an elliptic inclusion with a partially debonded region. The bi-material half space is divided into two parts along the vertical interface to investigate the two right angle plane respectively. Secondly, "image" method and conformal mapping method are employed to construct the scattering wave field in part I. Then the Green's function needed is obtained. Thirdly, with the aid of interface "conjunction" technique, a series of integral equations for determining the unknown force system could be set up through continuity conditions on the interface and Green's function. The method presented in this paper can be used to solve other similar scattering problem by arbitrary shape inclusion with partially debonded region.
\end{abstract}

\section{Introduction}

In the field of composite material, earthquake engineering and geotechnical engineering and other fields, debonded problems between elastic material are of theoretical and engineering application value.

Scattering problems of $\mathrm{SH}$ waves ${ }^{[1-7]}$ as the simplest problems in the research of scattering problem, have been studied many researchers, and many valuable results have been obtained. However, the scattering problems for some special structures are still unsolved.The bonding surface of different materials under physical or chemical injury will occur the phenomenon debonding, which will affect the mechanical and chemical properties. This paper mainly studies the effect on the mechanical properties.Many academics have studied the scattering of $\mathrm{SH}$ waves by structures with debonded region, but the mathematical model established are mainly concentrated in the whole space model or

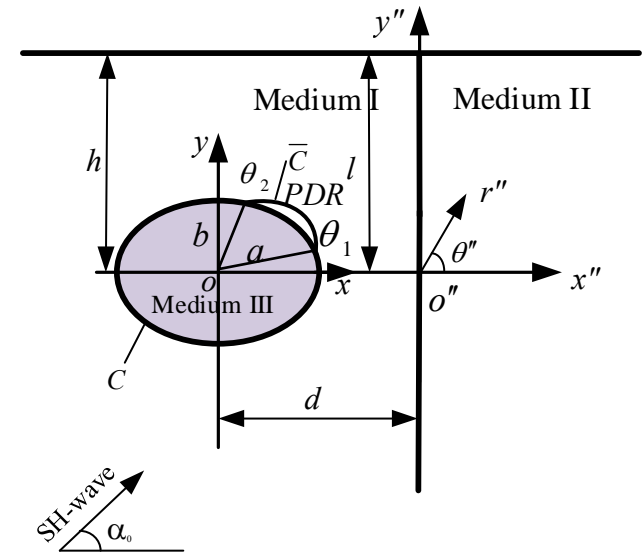

Fig.1 Elliptic inclusion with partially debonded region in bi-material half space homogeneous half space. The scattering of $\mathrm{SH}$ waves by elliptic inclusion with partially debonded region in bi-material half space are rarely investigated.

In this paper, complex function method and the idea of "conjunction" are empleed to study the scattering problems of SH waves by an elliptic inclusion near the vertical interface in bi-material half space.Image method and conformal mapping method are used to study the wave field outside the elliptic inclusion. In the elliptic domain, there are standing waves that satisfy the continuity conditions of displacement and stress on the boundary of domain I and domain III. And the stress continuity condition should be satisfied on the debonded region.

Fig.1 shows a bi-material half-space with an elliptical inclusion with partially dedondeb region (PDR) of half-macroaxis $a$ and half-brachyaxis $b$. The elliptic inclusion has material properties, shear modulus $\mu_{3}$ and mass body density $\rho_{3}$. The distances from the center of elliptical inclusion to the vertical interface and horizontal surface are $d$ and $h$, respectively. The incident angle of SH waves is $\alpha_{0}$. There are two coordinate systems setting on $O$ and $O^{\prime \prime}$ point. 


\section{Basic theory}

Conjunction method will be used in this paper, Green's function should be studied before. Green's function used in this paper is an essential solution of displacement field for a quarter-plane with an elliptical elastic inclusion by anti-plane harmonic line source $\delta\left(z-z_{0}\right)(z=x+\mathrm{i} y)$ loading at vertical surface. The displacement function $G$ must satisfy the governing equation with omitted the time harmonic factor $\exp (-\mathrm{i} \omega t)$ of the following form:

$$
\frac{\partial^{2} G}{\partial z \partial \bar{z}}+\frac{1}{4} k^{2} G=0
$$

In which, $z$ and $\bar{z}$ are the complex variables, $k=\omega / c_{s}$ is wave number, $\omega$ and $c_{s}=\sqrt{\mu / \rho}$ are the disturbing circular frequency and the shear velocity of the media, $\rho$ and $\mu$ are the mass body density and the shear modulus of the media, respectively.

The stresses corresponding to Eq.(1) can be written as:

$$
\tau_{r z}=\mu\left(\frac{\partial G}{\partial z} e^{\mathrm{i} \theta}+\frac{\partial G}{\partial \bar{z}} e^{-\mathrm{i} \theta}\right), \tau_{\theta z}=\mathrm{i} \mu\left(\frac{\partial G}{\partial z} e^{\mathrm{i} \theta}-\frac{\partial G}{\partial \bar{z}} e^{-\mathrm{i} \theta}\right)
$$

For wave scattering problems involving elliptical inclusion in the complex $(\mathrm{z}, \overline{\mathrm{z}})$ plane, it is possible to map the internal/external region of the elliptical inclusion into the inside/outside region of the circle(in the $(\eta, \bar{\eta})$ plane).

Introducing the mapping function:

$$
Z=\omega(\eta)=R\left(\eta+\frac{m}{\eta}\right), \eta=R e^{i \theta}
$$

The above mapping function map the outside of the inclusion in the $(\mathrm{z}, \overline{\mathrm{z}})$ plane into the region $|\eta|>1$. Consequently, the corresponding governing Eq.(1) in $(\eta, \bar{\eta})$ plane takes on the following form:

$$
\frac{1}{\omega^{\prime}(\eta) \overline{\omega^{\prime}(\eta)}} \frac{\partial^{2} G}{\partial \eta \partial \bar{\eta}}+\frac{1}{4} k^{2} G=0
$$

In $(\eta, \bar{\eta})$ plane, Eq.(2) can be written as:

$$
\tau_{r}=\frac{\mu}{R\left|\omega^{\prime}(\eta)\right|}\left(\eta \frac{\partial G}{\partial \eta}+\bar{\eta} \frac{\partial G}{\partial \bar{\eta}}\right), \quad \tau_{\theta}=\frac{\mathrm{i} \mu}{R\left|\omega^{\prime}(\eta)\right|}\left(\eta \frac{\partial G}{\partial \eta}-\bar{\eta} \frac{\partial G}{\partial \bar{\eta}}\right)
$$

\section{Anti-plane SH waves in domain I and domain II}

Considering the existence of the free boundary and the interface in bi-material media, the image method is employed to transform right angle space to full space. we can consider the full space model as an equivalent model with multiple wave sources. And the equivalent incident wave can be expressed as follows:

$$
\begin{aligned}
& W^{(i, e)}=W_{0} \exp \left\{\frac { \mathrm { i } k _ { 1 } } { 2 } \left[(\omega(\eta)-\mathrm{i} h) e^{-\mathrm{i} \alpha_{0}}+(\overline{\omega(\eta)}+\mathrm{i} h) e^{\mathrm{i} \alpha_{0}}+(\omega(\eta)-\mathrm{i} h-2 d) e^{-\mathrm{i} \gamma_{0}}\right.\right. \\
& \left.\left.+(\overline{\omega(\eta)}+\mathrm{i} h-2 d) e^{\mathrm{i} \gamma_{0}}\right]\right\}
\end{aligned}
$$

Where, $\gamma_{0}=\pi-\alpha_{0}, \alpha_{0}$ and $W_{0}$ are incident angle and the amplitude of the incident wave respectively.

Similarly, the equivalent reflected wave and refracted wave are:

$$
\begin{aligned}
& W^{(r, \mathrm{e})}=W_{1} \exp \left\{\frac { \mathrm { i } k _ { 1 } } { 2 } \left[(\omega(\eta)-i h) e^{-\mathrm{i} \alpha_{1}}+(\overline{\omega(\eta)}+\mathrm{i} h) e^{\mathrm{i} \alpha_{1}}+(\omega(\eta)-i h-2 d) e^{-\mathrm{i} \gamma_{1}}\right.\right. \\
& \left.\left.+(\overline{\omega(\eta)}+\mathrm{i} h-2 d) e^{\mathrm{i} \gamma_{1}}\right]\right\}
\end{aligned}
$$




$$
\begin{aligned}
& W^{(f, \mathrm{e})}=W_{2} \exp \left\{\frac { \mathrm { i } k _ { 2 } } { 2 } \left[(\omega(\eta)-\mathrm{i} h) e^{-\mathrm{i} \alpha_{2}}+(\overline{\omega(\eta)}+\mathrm{i} h) e^{\mathrm{i} \alpha_{2}}+(\omega(\eta)-\mathrm{i} h-2 d) e^{-\mathrm{i} \gamma_{2}}\right.\right. \\
& \left.\left.+(\overline{\omega(\eta)}+\mathrm{i} h-2 d) e^{\mathrm{i} \gamma_{2}}\right]\right\}
\end{aligned}
$$

In which, $\gamma_{1}=\pi-\alpha_{1}, \gamma_{2}=\pi-\alpha_{2}, \alpha_{1}$ and $\alpha_{2}$ are the reflection angle and refraction angle respectively.

\section{Standing waves in domain III}

Constructing standing wave in the elliptic domain need satisfy the stress free conditions on boundary $\bar{C}$ and continuity condition of displacement and stress on boundary $C$ :

$$
\tau_{r z}^{(\mathrm{st})}=\left\{\begin{array}{c}
0, \omega(\eta) \in \bar{C} \\
\frac{\mu_{3} k_{3} G_{0}}{2} \sum_{m=-\infty}^{\infty} C_{m}\left[J_{m-1}\left(k_{3}|\omega(\eta)|-J_{m+1}\left(k_{3}|\omega(\eta)|\right)\right]\left[\frac{\omega(\eta)}{|\omega(\eta)|}\right]^{m}, \omega(\eta) \in C\right.
\end{array}\right.
$$

In which, $C_{m}$ is unknown coefficient, $W_{T 0}$ is displacement amplitude of standing wave, $W_{T 0}=1$ 。

Standing waves in elliptic domain:

$$
W^{(\mathrm{st})}=W_{T 0} \sum_{m=-\infty}^{\infty} D_{n} J_{n}\left(k_{3}|\omega(\eta)|\right)\left[\frac{\omega(\eta)}{\mid \omega(\eta)}\right]^{n}
$$

In which, $D_{m}$ is unknown coefficient.

The Fourier expansion of Eq.(10) within the range $[-\pi, \pi]$ can be written as

$$
\tau_{r z}^{(\mathrm{st})}=\frac{\mu_{3} k_{3} W_{T 0}}{2} \sum_{n=-\infty}^{\infty} \sum_{m=-\infty}^{\infty} C_{m} a_{m n}\left[J_{m-1}\left(k_{3}|\omega(\eta)|-J_{m+1}\left(k_{3}|\omega(\eta)|\right)\right]\left[\frac{\omega(\eta)}{|\omega(\eta)|}\right]^{n}\right.
$$

In which:

$$
a_{m n}=\left\{\begin{array}{cl}
\frac{\theta_{1}-\theta_{2}+2 \pi}{2 \pi} & , m=n \\
\frac{e^{\mathrm{i}(\mathrm{m}-\mathrm{n}) \theta_{1}}-e^{\mathrm{i}(\mathrm{m}-\mathrm{n}) \theta_{2}}}{2 \pi i(\mathrm{~m}-\mathrm{n})} & , m \neq n
\end{array}\right.
$$

In coordinate $(\eta, \bar{\eta}), \theta_{1}$ and $\theta_{2}$ are the start angel and end angel of debonded region.

Comparing Eq. (11) with the stress of Eq. (10) under the condition $|\eta|=1$

$$
D_{n}=G_{0} \sum_{m=-\infty}^{\infty} C_{m} \frac{\left.J_{m-1}\left|k_{3} \omega(\eta)\right|_{|\eta|=1}\left|-J_{m+1}\right| k_{3} \omega(\eta)\right|_{|\eta|=1} \mid}{\left.J_{n-1}\left|k_{3} \omega(\eta)\right|_{|\eta|=1}\left|-J_{n+1}\right| k_{3} \omega(\eta)\right|_{|\eta|=1} \mid} a_{m n}
$$

The standing wave field in domain II can be expressed as:

$$
G^{\text {(st) }}=W_{0} \sum_{n=-\infty}^{\infty} \sum_{m=-\infty}^{\infty} C_{m} \frac{\left.J_{m-1}\left|k_{3} \omega(\eta)\right|_{|\eta|=1}\left|-J_{m+1}\right| k_{3} \omega(\eta)\right|_{|\eta|=1} \mid}{\left.J_{n-1}\left|k_{3} \omega(\eta)\right|_{|\eta|=1}\left|-J_{n+1}\right| k_{3} \omega(\eta)\right|_{|\eta|=1} \mid} a_{m n} J_{n}\left(k_{2}|\omega(\eta)|\right)\left[\frac{\omega(\eta)}{|\omega(\eta)|}\right]^{n}
$$

The continuity condition on the boundary of domain III and domain I

$$
\left\{\begin{array}{l}
W^{(\mathrm{i}, \mathrm{e})}+W^{(r, e)}+W^{(\mathrm{s})}=W^{(\mathrm{st})}, \quad \theta \in C \\
\tau_{r z}^{(i, \mathrm{e})}+\tau_{r z}^{(\mathrm{r}, \mathrm{e})}+\tau_{r z}^{s}=\tau_{r z}^{(\mathrm{st})}, \theta \in(-\pi, \pi)
\end{array}\right.
$$

The Green function we need can be get by solved Eq.(15). 


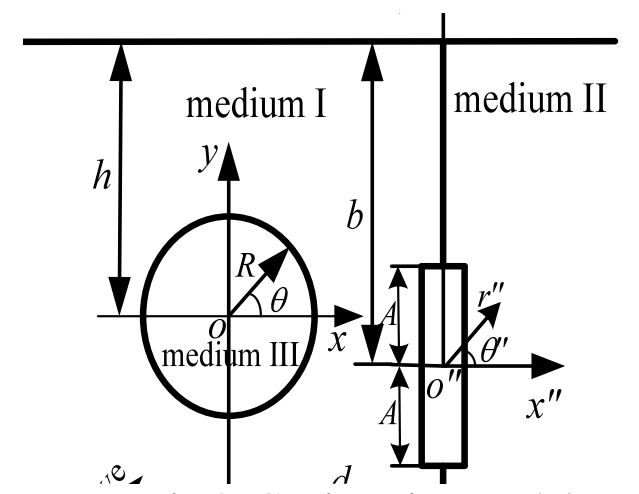

Fig 2. Conjunction model

As shown in Fig.2, unknown force systems $f_{1}$ and $f_{2}$ are loaded on the conjunction section to satisfy the continuity conditions on the interface. So a series of Fredholm integral equations for determining the unknown forces can be set up .

The total displacements $W^{(\mathrm{I})}, W^{(\mathrm{II})}$ and total stresses $\tau_{\theta^{\prime \prime} z^{\prime \prime}}^{(\mathrm{I})}, \tau_{\theta^{\prime \prime} z^{\prime \prime}}^{(\mathrm{II})}$ in the two parts are:

$$
\left\{\begin{array}{l}
W^{(\mathrm{I})}=W^{(i, e)}+W^{(r, e)}+W^{(s)}, \quad W^{(\mathrm{II})}=W^{(f, e)} \\
\tau_{\theta^{\prime \prime} z^{\prime \prime}}^{(\mathrm{I})}=\tau_{\theta^{\prime \prime} z^{\prime \prime}}^{(i, e)}+\tau_{\theta^{\prime \prime} z^{\prime \prime}}^{(r, e)}+\tau_{\theta^{\prime \prime} z^{\prime \prime}}^{(s)}, \quad \tau_{\theta^{\prime} z^{\prime \prime}}^{(\mathrm{II})}=\tau_{\theta^{\prime \prime} z^{\prime \prime}}^{(f, e)}
\end{array}\right.
$$

Where, $W^{(s)}$ is the displacement of the scattering wave. $\tau_{\theta z}^{(s)}$ is the stress of the scattering wave.

The stress continuity condition can be expressed as:

$$
\tau_{\theta^{\prime \prime} z^{\prime \prime}}^{(\mathrm{I})} \sin \theta_{0}^{\prime \prime}+f_{1}\left(r_{0}^{\prime \prime}, \theta_{0}^{\prime \prime}\right)=\tau_{\theta^{\prime \prime} z^{\prime \prime}}^{(\mathrm{II})} \sin \theta_{0}^{\prime \prime}+f_{2}\left(r_{0}^{\prime \prime}, \theta_{0}^{\prime \prime}\right)
$$

Where $r_{0}^{\prime \prime}$ and $\theta_{0}^{\prime \prime}$ are the polar coordinates in the global coordinate system $x^{\prime \prime} o^{\prime \prime} y^{\prime \prime}$ and $z^{\prime \prime}=r^{\prime \prime} \exp \left(\mathrm{i} \theta^{\prime \prime}\right), z^{\prime \prime}=z-d$. when $\theta_{0}^{\prime \prime}=\beta_{1}=-\pi / 2,0 \leq r_{0}^{\prime \prime} \leq \infty$, when $\theta_{0}^{\prime \prime}=\beta_{2}=\pi / 2,0 \leq r_{0}^{\prime \prime} \leq h$.

According to $\tau_{\theta^{\prime \prime} z^{\prime \prime}}^{(i, e)}+\tau_{\theta^{\prime \prime} z^{\prime \prime}}^{(r, e)}=\tau_{\theta^{\prime \prime} z^{\prime \prime}}^{(f, e)}$, we can get:

$$
f_{1}\left(r_{0}^{\prime \prime}, \theta_{0}^{\prime \prime}\right)=f_{2}\left(r_{0}^{\prime \prime}, \theta_{0}^{\prime \prime}\right), \theta_{0}=\beta_{1}, \beta_{2}
$$

The displacement continuity conditions can be written as:

$$
W^{(\mathrm{I})}+W^{\left(f_{1}\right)}+W^{\left(c_{1}\right)}=W^{(\mathrm{II})}+W^{\left(f_{2}\right)}+W^{\left(c_{2}\right)}
$$

According to $W^{(i, e)}+W^{(r, e)}=W^{(f, e)}$, we can obtain:

$$
W^{(\mathrm{s})}+W^{\left(f_{1}\right)}=W^{\left(f_{2}\right)}
$$

Where, $W^{\left(f_{1}\right)}$ is the displacement field caused by force system $f_{1}$, and $W^{\left(f_{2}\right)}$ is the displacement field caused by force system $f_{2}$.

According to the continuity condition and the Green's function we have obtained, the integral equations with unknown anti-plane forces can be expressed as:

$$
\begin{aligned}
& \int_{A}^{l} f_{1}\left(r_{0}^{\prime \prime}, \beta_{2}\right)\left[G_{1}\left(r^{\prime \prime}, \beta_{1} ; r_{0}^{\prime \prime}, \beta_{2}\right)+G_{2}\left(r^{\prime \prime}, \beta_{1} ; r_{0}^{\prime \prime}, \beta_{2}\right)\right] d r_{0}^{\prime \prime}+ \\
& \int_{A}^{\infty} f_{1}\left(r_{0}^{\prime \prime}, \beta_{1}\right)\left[G_{1}\left(r^{\prime \prime}, \beta_{1} ; r_{0}^{\prime \prime}, \beta_{1}\right)+G_{2}\left(r^{\prime \prime}, \beta_{1} ; r_{0}^{\prime \prime}, \beta_{1}\right)\right] d r_{0}^{\prime \prime}=\left[-\mathrm{W}^{(\mathrm{S})}\right]_{\theta_{0}^{\prime \prime}=\beta_{1}} \\
& \int_{A}^{l} f_{1}\left(r_{0}^{\prime \prime}, \beta_{2}\right)\left[G_{1}\left(r^{\prime \prime}, \beta_{2} ; r_{0}^{\prime \prime}, \beta_{2}\right)+G_{2}\left(r^{\prime \prime}, \beta_{2} ; r_{0}^{\prime \prime}, \beta_{2}\right)\right] d r_{0}^{\prime \prime}+ \\
& \int_{A}^{\infty} f_{1}\left(r_{0}^{\prime \prime}, \beta_{1}\right)\left[G_{1}\left(r^{\prime \prime}, \beta_{2} ; r_{0}^{\prime \prime}, \beta_{1}\right)+G_{2}\left(r^{\prime \prime}, \beta_{2} ; r_{0}^{\prime \prime}, \beta_{1}\right)\right] d r_{0}^{\prime \prime}=\left[-\mathrm{W}^{(\mathrm{S})}\right]_{\theta_{0}^{\prime \prime}=\beta_{2}}
\end{aligned}
$$

In which, $G_{1}$ and $G_{2}$ are the Green's functions in domain I and II respectively.

Hoop stress around the inclusion can be expresses as

$$
\tau_{\theta z}^{(\bullet)}=\tau_{\theta z}^{(\mathrm{l})}+\int_{A}^{l} f_{1}\left(r_{0}^{\prime \prime}, \beta_{1}\right) \frac{\mu_{1}}{r} \frac{\partial G_{1}\left(r^{\prime \prime}, \theta ; r_{0}^{\prime \prime}, \beta_{2}\right)}{\partial \theta} d r_{0}^{\prime \prime}+\int_{A}^{\infty} f_{1}\left(r_{0}^{\prime \prime}, \beta_{2}\right) \frac{\mu_{1}}{r} \frac{\partial G_{1}\left(r^{\prime \prime}, \theta ; r_{0}^{\prime \prime}, \beta_{2}\right)}{\partial \theta} d r_{0}^{\prime \prime}
$$

In which, $\tau_{\theta z}^{(\mathrm{I})}=\tau_{\theta z}^{(i, \mathrm{e})}+\tau_{\theta z}^{(r, \mathrm{e})}+\tau_{\theta z}^{(s)}$ 。

\section{Conclusion}

In this paper, only theory deduction is presented, analitical results will be given in the follow-up work.

\section{References}

[1] Lee V W, Manoogian M E. Surface motion above an arbitrary shape underground cavity for incident SH wave[J]. European Earthquake Engineering, 1995, 8(1): 3-11. 
[2] Lee V W, Amornwongpaibun A. Scattering of anti-plane ( $\mathrm{SH}$ ) waves by a semi-elliptical hill: I-Shallow hill[J]. Soil Dynamics and Earthquake Engineering, 2013, 53: 116-125

[3] LUO Hao, V W Lee, LIANG Jian-wen. Anti-plane(SH) waves diffraction by an underground semi-circular cavity: analytical solution[J]. Earthquake engineering and engineering vibration, 2010,9(3): 385-396.

[4] QI Hui, YANG Jie. Dynamic analysis for circular inclusion of arbitrary positions near interfacial crack impacted by SH-wave in half-space[J]. European Journal of Mechanics /A Solids, 2012, 36: 18-24.

[5] QI Hui,YANG Jie,SHI Yong,TIAN Jia-yong. Dynamic Analysis for Circular Inclusion near Interfacial Crack Impacted by SH-Wave in Half Space[J]. Journal of Mechanics, 2012, 28(1): 143-151.

[6] YANG Zai-lin, YAN Peilei, LIU Dian-kui. Scattering of SH-waves and ground motion by an elastic cylindrical inclusion and a crack in half space[J]. Chinese Journal of Theoretical and Applied Mechanics, 2009, 41(2): 229-235.(in Chinese)

[7] Jiaxi Zhao, Hui Qi, Diankui Lu, Hongliang Li. Scattering of SH waves by a shollow buried circular liner with a partially debonded region[J]. CHINESE JOU RNAL OF SOLID MECHANICS, 2008,29(3):301-306(in Chinese) 